\title{
Métodos qualitativos de pesquisa aplicados aos estudos da agricultura familiar
}

\section{Juliano Luiz Fossá ${ }^{1}$ e Leonel Piovezana ${ }^{2}$}

1 Doutorando em Administração - UFSC, Mestre em Políticas Sociais e Dinâmicas Regionais Universidade Comunitária da Região de Chapecó - UNOCHAPECÓ, Brasil. E-mail: j.fossa@unochapeco.edu.br

2 Doutor em Desenvolvimento Regional - UNISC. Docente do Programa Stricto Sensu em Educação da Unohapecó, Brasil. E-mail: leonel@unochapeco.edu.br

RESUMO: Este artigo apresenta uma análise dos métodos qualitativos de pesquisa utilizados em teses publicadas pelo Programa de Pós-Graduação em Desenvolvimento Rural da Universidade Federal do Rio Grande do Sul (PGDR/UFRGS) em temáticas referentes aos estudos da agricultura familiar. Ressaltamos as características dos métodos qualitativos, bem como sua importância nas investigações científicas e compreensões da realidade social. Selecionamos 12 teses que abordam a temática da agricultura familiar e se utilizam de métodos qualitativos de pesquisa no quadriênio compreendido entre 2013-2016. As análises apontaram que na totalidade dos trabalhos a principal estratégia do método materializou-se pela realização de entrevistas, compreendida como técnica para coleta de dados. Quanto à forma, em $100 \%$ (cem por cento) das teses, as entrevistas foram semiestruturadas e possibilitaram oportunidades e interações de conhecimentos nos momentos de suas realizações. Ressaltamos que houve utilização de outras técnicas para a coleta de dados, que ajudaram na assimilação e compreensão das informações de campo, como por exemplo: informantes-chave e anotações em diários de campo. As técnicas para análise dos dados coletados foram a partir de conteúdo por meio de categorias de análise. Consideramos que a utilização dos métodos qualitativos são aspectos positivos, pois ressaltam a importância social, a diversidade e complexidade que integram esta categoria. Concluímos que a diversidade encontrada nas teses analisadas permitem inúmeras possibilidades de investigações sociais sobre a agricultura familiar por meio do método qualitativo. Nessa perspectiva de olhares, percebemos que o método qualitativo, assume também, uma perspectiva multifacetária ao se apresentar como caminho metodológico adequado de investigações sociais.

Palavras-chave: Pesquisa. Agricultura. Familiar. Métodos Qualitativos.

\section{Qualitative research methods applied to studies of family farming}

ABSTRACT: This paper presents an analysis of the qualitative research methods used in theses published by the Post-graduate Program in Rural Development of the Federal University of Rio Grande do Sul (PGDR / UFRGS) in themes related to family farming studies. We highlight the characteristics of qualitative methods, as well as their importance in scientific investigations and understandings of social reality. We chose 12 theses that approach the theme of family farming and use qualitative research methods in the quadrennium between 2013-2016. The analyzes indicated that in all the 
works the main strategy of the method was carried out by interviews, understood as a technique for data collection. Regarding the form, in $100 \%$ (one hundred percent) of theses, the interviews were semi-structured and enabled opportunities and interactions of knowledge at the moment of their achievements. We emphasize the use of other techniques for data collection, which helped in the assimilation and understanding of field information, such as: key informants and notes in field journals. The techniques for analyzing the collected data were from content through analysis categories. We consider that the use of qualitative methods are positive aspects, since they emphasize the social importance, the diversity and complexity that integrate this category. We conclude that the diversity found in the analyzed theses allows numerous possibilities of social investigations on family farming through the qualitative method. From this perspective, we notice that the qualitative method also assumes a multifaceted perspective when presenting itself as an appropriate methodological journey of social investigations.

Keywords: Research. Family Farming. Qualitative Methods.

\section{INTRODUÇÃO}

Nos últimos cinquenta anos, os métodos qualitativos de pesquisa vêm se afirmando como alternativa aos métodos tradicionais, possibilitando aos pesquisadores sociais diversas maneiras de investigações enquanto método. A abordagem qualitativa permite a proximidade entre o pesquisador e os sujeitos de pesquisa, admitindo o próprio pesquisador como parte do processo de investigação.

O método qualitativo, em linhas gerais, procura entender o específico, busca a intensidade e a profundidade do fenômeno que se propõe a pesquisar. Essas características do método (como inúmeras outras) têm chamado à atenção, cada vez mais, de pesquisadores, que se desafiam por meio dessa abordagem nos processos de construção do conhecimento através de pesquisas científicas. Entre estes pesquisadores, naturalmente estão àqueles interessados na temática da agricultura familiar.

A agricultura familiar se constitui em uma temática de estudo com amplas possibilidades e olhares sobre a realidade social. Os estudos sobre a agricultura familiar têm se difundido nas mais diversas áreas do conhecimento e, consequentemente, contribuído significativamente para o avanço da ciência nesse campo.

É neste contexto que este trabalho se situa, pois se propõe a apresentar quais métodos qualitativos de pesquisa têm sido utilizados nos estudos e pesquisas avançadas que envolvam a agricultura familiar. Como delimitação desta pesquisa, optou-se em investigar tal aspecto, através do Programa de Pós-Graduação em Desenvolvimento Rural (PGDR) da Universidade Federal do Rio Grande do Sul (UFRGS), que possui longa tradição de estudos sobre o meio rural e a agricultura. O PGDR possui atualmente o conceito 6 na avaliação da CAPES (área interdisciplinar) e é reconhecido nacionalmente e internacionalmente pela sua qualidade acadêmica.

O objetivo deste estudo é analisar os métodos qualitativos de pesquisa utilizados em teses publicadas pelo PGDR/UFRGS em temáticas referente à agricultura familiar. A realiza- 
ção desta pesquisa se justifica pelo fato da temática escolhida possuir significativa relação com a tese a ser desenvolvida ao longo do doutoramento do primeiro autor, bem como a importância acadêmica de estudos que envolvam a construção do conhecimento por meio de pesquisas de abordagem qualitativa.

Este trabalho está organizado em quatro seções, além desta introdução. Na seção dois são apresentados referenciais teóricos em relação ao método qualitativo de pesquisa, além de breve conceitualização da agricultura familiar enquanto segmento social e econômico. Na seção três são descritos os aspectos do percurso metodológico realizado nesta pesquisa. A seção quatro apresenta as análises realizadas em relação aos métodos utilizados nas 12 teses selecionadas e, por fim, são tecidas as considerações finais.

\section{MÉTODOS QUALITATIVOS DE PESQUISA}

Na construção deste estudo, parte-se da compreensão que a utilização métodos qualitativos de pesquisa são uma alternativa nos processos de investigação e pesquisa científica. Neste sentido é dado como ultrapassada a visão de que os métodos qualitativos de pesquisa podem ser classificados como inferiores aos quantitativos ou ainda de "segunda linha" (BRAYMAN, 1988). Em suporte a esta afirmação, Godoi e Balsini (2007, p. 89) apontam que essa discussão "[...] torna-se insipiente, não apenas pelo seu caráter simplista mas, fundamentalmente, porque, entre métodos, não se trata de uma mera escolha voluntária do pesquisador." Superada essa questão, pretendemos apresentar aspectos introdutórios da utilização dos métodos qualitativos.

Para Demo (1995), os métodos qualitativos de pesquisa procuram andar na contramão do desgastado percurso metodológico tradicional, ou seja, se distanciam da supremacia do método e procuram compreender a realidade social na sua totalidade e complexidade, sua evolução histórica para em momento posterior adequar essa realidade ao método adequado. $\mathrm{O}$ método qualitativo deve ser entendido como um instrumento, um caminho, para construção da crítica social (SCHWANDT, 2006).

É nessa perspectiva que os estudos qualitativos se distanciam do paradigma positivista, pois admitem como concepção uma série de características que são rejeitadas por este modo de construção de conhecimento. A primeira característica é a aceitação da subjetividade como elemento inerente às investigações sociais (LAPERRIÈRE, 2008). Uma segunda característica é o fato da interação do pesquisador com os sujeitos, ou seja, não há um distanciamento no processo de observação e coleta de dados. Outra característica é a profundidade, a intensidade das investigações dos fenômenos sociais que o método está disposto a compreender, o foco é o específico, não tendo como preocupação a generalização.

Godoy (1995, p. 58), de maneira geral, define que a pesquisa qualitativa como aquela que,

[...] não procura enumerar e/ou medir os eventos estudados, nem emprega instrumental estatístico na análise dos dados. Parte de questões ou focos de interesses amplos, que vão se definindo à medida que o estudo se desenvolve. Envolve a obtenção de dados descritivos sobre pessoas, lugares e 
processos interativos pelo contato direto do pesquisador com a situação estudada, procurando compreender os fenômenos segundo a perspectiva dos sujeitos, ou seja, dos participantes da situação em estudo.

No mesmo sentido, Vieira (2004) enfatiza que uma das características fundamentais das pesquisas qualitativas é a não utilização de instrumental estatístico na análise de dados. Contudo, essa condição de não utilização de modo algum significa qualquer impedimento na aproximação e utilização dos métodos quali-quanti de forma conjunta. Segundo Strauss e Corbin (2008), a interação dos métodos, quando adequada, pode possibilitar avanços em termos metodológicos, proporcionando suplementariedade, complementariedade nas investigações científicas. Ainda conforme Vieira (2004, p. 18), o método qualitativo oferece possibilidades de "[...] um maior grau de flexibilidade ao pesquisador para adequação da estrutura teórica ao estudo do fenômeno [...]".

Denzin e Lincoln (2006) caracterizam o "fazer" do método qualitativo como sendo amplo e de caráter de "confeccionador" de colchas. Em outras palavras, podemos relacionar a um trabalho artesanal, realizado com muito cuidado, que por sua vez exige um rigoroso cuidado a todos os detalhes. Para Strauss e Corbin (2008, p. 24), a análise qualitativa refere-se "[...] ao processo não-matemático de interpretação, feito com objetivo de descobrir conceitos e relações nos dados brutos e de organizar esses conceitos e relações em um esquema explanatório teórico."

Alves-Mazzotti (2001) aponta que as pesquisas qualitativas permitem o pesquisador um maior grau de abertura, pois é ao longo do percurso da pesquisa que se define tanto a estrutura, quanto o método mais adequado à investigação que está realizando.

Em relação ao planejamento da pesquisa sob a perspectiva dos métodos qualitativos, assim como mencionamos acima, em geral, a estrutura, o design do projeto de pesquisa não necessariamente precisa estar definido e fixado antes da imersão do pesquisador no campo (ALVES-MAZZOTTI, 2001).

É importante lembrar também que esse planejamento não precisa e nem deve ser apriorístico no sentido mais estrito, pois, nos estudos qualitativos, a coleta sistemática de dados deve ser precedida por uma imersão do pesquisador no contexto a ser estudado. Essa fase exploratória permite que o pesquisador, sem descer ao detalhamento exigido na pesquisa tradicional, defina pelo menos algumas questões iniciais bem como os procedimentos adequados a investigações dessas questões. (ALVES-MAZZOTTI, 2001, p. 48).

Um aspecto importante é de que quanto mais aberto o planejamento em termos de estrutura, mais desafiador se tornar ao pesquisador. Nesse sentido, é viável que em situações nas quais o pesquisador não seja muito experiente que se adote, previamente, pelo menos uma estrutura mínima do planejamento da pesquisa. Para Alves-Mazzotti (2001), outro aspecto importante em relação ao plano de pesquisa, especialmente aos estudantes, é que os projetos de pesquisa necessariamente precisam passar por aprovações em órgãos institucionais, como os conselhos de ética em pesquisa.

Os estudos qualitativos são dotados de inúmeros critérios de cientificidade, sendo as 
posições em contrário amplamente superadas entre pesquisadores no meio acadêmico, como nos apresenta Godoi e Balsini (2007) ao referenciarem um conjunto de artigos científicos que criticam posições em contrário à cientificidade dos métodos qualitativos. Neste sentido, Laperrière (2008) é um exemplo de construção teórica que nos fornece um amplo conjunto de procedimentos que asseguram a afirmação sobre critérios de cientificidade nos métodos qualitativos. Entre os elementos apontados pelo autor estão: validade interna; validade externa; e confiabilidade.

Na concepção de Vergara (2015), é responsabilidade do pesquisador a escolha do método adequado para cada investigação científica. Essa escolha não necessariamente precisa ser realizada ex ante, como já mencionamos acima, mas no decorrer do seu caminho, de seu percurso de pesquisa de campo. Entre as inúmeras possibilidades para investigação pela abordagem qualitativa, a própria Vergara (2015) em seu livro Métodos de Pesquisa em Administração apresenta uma síntese conceitual de 22 métodos de pesquisa qualitativa.

Neste sentido, percebe-se a diversidade de maneiras de métodos qualitativos nas investigações científicas de um modo geral e também no campo dos estudos organizacionais. $O$ objetivo da presente seção foi apresentar ao leitor aportes iniciais, bem como características da utilização de métodos qualitativos de pesquisa. No próximo item demonstramos conceitos sobre a categoria da agricultura familiar, assim como justificar a importância de estudos nessa temática.

\subsection{Estudos sobre agricultura familiar: uma categoria de análise}

Os referenciais teóricos há muitos anos classificam e distinguem a agricultura brasileira na dualidade de agricultura patronal/empresarial e agricultura familiar, sendo que neste estudo centralizaremos a análise na agricultura familiar. Mattei (2006) aponta que esses agricultores familiares também são designados como pequenos produtores, produtores familiares, produtores de baixa renda ou agricultores de subsistência. Entretanto, parte-se do entendimento que os conceitos se alteram com o passar dos anos. Conforme apontado por Seyferth (2011, p. 397), "as definições são ferramentas do pensamento e não verdades que duram para sempre".

Para Guanziroli (2007), uma das possibilidades para classificar a agricultura familiar é a partir das formas e organização social do seu processo produtivo, que essencialmente se caracterizam pelas seguintes aspectos: mão de obra familiar; maior parte da renda advinda do trabalho familiar na propriedade; produção diversificada; e estabelecimentos rurais familiares. Por isso, em muitas situações, a delimitação de tipos de agricultura serve ao propósito de oferecer programas e políticas específicas para determinada categoria.

Para Wanderley (2001), a agricultura familiar pode ser compreendida como aquela em que a família é a proprietária dos meios de produção e ao mesmo tempo assume o trabaIho na propriedade rural, apresentando, assim, uma significativa diversidade nas formas sociais de organização. Na visão de Abramovay (2003), a agricultura familiar apresenta multiplicidade de atores sociais (que vão muito além da produção agropecuária), os quais são sustentados pelos laços sociais individuais e de suas organizações. 
Moreira (2013) afirma que as definições das expressões da agricultura familiar se constituem em elementos criteriosos de identificação dos sujeitos analisados e de seus territórios, seu contexto social, econômico e cultural. No entendimento de Schneider (2009), os estudos da agricultura familiar exigem análises sociológicas de múltiplas dimensões sobre a ampla diversidade de suas formais sociais.

Os estudos sobre a agricultura familiar se justificam e assumem expressivos significados, pois a análise dessa categoria, sua dicotomia com a grande propriedade e as relações com a sociedade vão muito além da questão produtiva (MATTEI, 2015). A esse respeito, Costa (2013) ratifica essa condição afirmando que o modo de vida e as relações sociais da agricultura familiar não podem ser explicados meramente por estatísticas.

O debate sobre Desenvolvimento Rural no Brasil tem sido configurado de diversas formas e perante distintos focos. Embora as diversas abordagens mobilizadas para se explicar e orientar os cursos desse debate, a redução das desigualdades sociais e a promoção de vida mais digna no campo são metas e indicadores recorrentemente mobilizados no meio acadêmico e político para se discutir resultados de processos de Desenvolvimento. (SILVA, 2015, p. 22).

Em uma perspectiva de conceituação institucional, a Lei n. 11.326/2006 classifica a categoria da agricultura familiar como sendo aquela que: i) não detenha, a qualquer título, área maior do que quatro módulos fiscais; ii) utilize predominantemente mão de obra da própria família nas atividades econômicas do seu estabelecimento ou empreendimento; iii) tenha renda familiar predominantemente originada de atividades econômicas vinculadas ao próprio estabelecimento ou empreendimento; iv) dirija seu estabelecimento ou empreendimento com sua família (BRASIL, 2006).

Neste sentido, as análises teóricas explicativas em relação à agricultura familiar devem ser desenvolvidas a partir de um olhar de várias faces e perspectivas. Entre estas perspectivas estão modo de vida, reprodução social, sucessão familiar, papel do Estado, produção, relações com o mercado capitalista, entre outros inúmeros temas fundamentais para o entendimento desse importante segmento social. É neste contexto que se legitima e se justifica a importância de pesquisas avançadas na temática e, inclusive, na forma que este conhecimento vem sido construído, especialmente os vinculados aos programas de PósGraduação Stricto Sensu.

\section{PERCURSO METODOLÓGICO}

A aproximação com a realidade leva a produzir conhecimentos a partir de experiências em diferentes espaços e contextos sócios históricos. Em se tratando do conhecimento científico, esse aperfeiçoa o uso da racionalidade ao propor uma forma sistemática, metódica e crítica de desvelar e explicar a realidade (KOCHE, 2002). Bocchi (2004, p. 54) refere que o "[...] método designa um caminho pelo qual se obtém certo resultado. Pode designar também um conjunto de procedimentos e de regras utilizadas para atingir um objeto desejado". 
Nessa mesma perspectiva, Minayo (2012, p. 300) afirma que “[...] métodos e instrumentos são caminhos mediadores para permitir ao pesquisador o aprofundamento de sua pergunta central e suas perguntas sucessivas, levantadas a partir do encontro com seu objeto empírico ou documental".

A delimitação deste estudo contempla teses que se utilizaram de métodos qualitativos de pesquisa publicados sobre a temática da agricultura familiar no período de 2013 a 2016 pelo PGDR/UFRGS; neste sentido, foram selecionadas 12 teses. A escolha por este programa justifica-se devido ao PGDR ser um centro de excelência no país em estudos e pesquisas referentes à agricultura familiar, amplamente reconhecido por sua qualidade acadêmica.

\begin{tabular}{|c|c|c|}
\hline Ordem & Título Tese & Ano de publicação \\
\hline 1 & $\begin{array}{l}\text { Sucessão Hereditária na Agricultura Familiar: Valores, Motivações e Influências que Orientam as } \\
\text { Decisões dos Atores }\end{array}$ & 2016 \\
\hline 2 & $\begin{array}{l}\text { Organizações Sociais e Políticas Públicas: Inserção da FETRAF-SUL nas Políticas Públicas de Segu- } \\
\text { rança Alimentar e Nutricional }\end{array}$ & 2016 \\
\hline 3 & $\begin{array}{l}\text { Produzindo Prerrogativas de Cidadania: o Acesso da Agricultura Familiar à Política de Forneci- } \\
\text { mento de Produtos para a Alimentação Escolar }\end{array}$ & 2015 \\
\hline 4 & $\begin{array}{l}\text { Os Processos de Inovação e as Interações nas Agroindústrias Familiares em Regiões do Brasil e } \\
\text { da Itália }\end{array}$ & 2015 \\
\hline 5 & $\begin{array}{l}\text { Vulnerabilidade e Resiliência Social da Agricultura Familiar do Alto Guaporé (MT) no Contexto } \\
\text { das Mudanças Ambientais Contemporâneas }\end{array}$ & 2015 \\
\hline 6 & $\begin{array}{l}\text { Mudanças Institucionais na Agricultura Familiar: as Políticas Locais e as Políticas Públicas nas } \\
\text { Trajetórias das Famílias nas Atividades de Processamento de Alimentos no Rio Grande do Sul }\end{array}$ & 2014 \\
\hline 7 & $\begin{array}{l}\text { A Qualificação e a Formação da Identidade Profissional dos Agricultores Familiares da Região de } \\
\text { Januária - MG. }\end{array}$ & 2013 \\
\hline 8 & Racionalidades modernas e identidades socioprofissionais de agricultores familiares & 2013 \\
\hline 9 & $\begin{array}{l}\text { Mulheres na agricultura familiar do semiárido Norte - Mineiro: divisão social do trabalho e } \\
\text { gênero no Projeto Jaíba }\end{array}$ & 2013 \\
\hline 10 & $\begin{array}{l}\text { Educação do campo e docência no contexto da agricultura familiar: o Programa Escola Ativa } \\
\text { (PEA/MEC) no município de Salinas - MG }\end{array}$ & 2013 \\
\hline 11 & $\begin{array}{l}\text { Reprodução social e práticas socioprodutivas de agricultores familiares na microrregião de } \\
\text { Altamira, Pará, Brasil. }\end{array}$ & 2013 \\
\hline 12 & $\begin{array}{l}\text { Interfaces e cogestão nas políticas para agricultura familiar - uma análise do Programa Nacional } \\
\text { de Habitação Rural. }\end{array}$ & 2013 \\
\hline
\end{tabular}

Fonte: elaboração do autor (2017), a partir de dados do PGDR/UFRGS.

Este estudo se baseia numa abordagem qualitativa apoiando-se no levantamento bibliográfico, levando em conta: (a) levantamento de referências; (b) leituras; (c) registro das informações; (d) a estruturação escrita do texto, que, para Dmitruk Ortiz (2012, p. 47), "[...] consiste num conjunto sequencial de procedimentos planejados para recolher, analisar e sintetizar as principais contribuições teóricas sobre um assunto, assim como para elaborar um texto escrito sobre um objeto de estudo."

Após a realização da seleção das teses, procedeu-se a análise em relação ao conjunto de métodos qualitativos utilizados no conjunto de trabalhos. A organização e estruturação da seção a seguir se deu de forma descritiva, ou seja, elencando os principais aspectos utilizados pelos autores ao longo de seus percursos metodológicos. 


\section{ANÁLISE DOS MÉTODOS QUALITATIVOS}

Nesta seção apresentamos as análises descritivas realizadas em relação à utilização dos métodos qualitativos em 12 teses que abordaram temáticas relacionadas à agricultura familiar no quadriênio 2013-2016. Estas teses estão vinculadas ao PGDR/UFRGS que, conforme mencionamos na introdução e seção anterior deste trabalho, se constitui em um centro de excelência em estudos e pesquisas sobre desenvolvimento rural e consequentemente agricultura familiar.

A construção da análise baseou-se no objetivo desta pesquisa e foi construída em relação à verificação de quais métodos qualitativos foram utilizados no conjunto de teses selecionadas para realização deste estudo. A partir de agora, com objetivo de facilitarmos a leitura, denominaremos por "Tese 01 , Tese 02 Tese $03, . .$. Tese 12 " e assim por diante, conforme Quadro 1.

No processo de busca pelos métodos que foram utilizados pelos autores das referidas teses, iniciou-se pela identificação da caracterização de cada trabalho. Nesse sentido, verificou-se que, das 12 teses pesquisadas, oito delas se caracterizam pela abordagem qualitativa, entretanto, duas delas (Tese 03 e Tese 05 ) não descreveram textualmente essa condição, mas pelos procedimentos metodológicos descritos foi identificada essa condição. As outras quatro teses (Tese 01, Tese 02, Tese 04 e Tese 08) se utilizaram da abordagem qualitativa e também da quantitativa. Essa triangulação, esses complementos entre os métodos é um aspecto interessante e até elogiável, pois permite maiores possibilidades nas investigações científicas.

Outra característica inicial que se torna importante revelar diz respeito à disposição dos procedimentos metodológicos na estrutura e/ou desenho das teses. Esses procedimentos foram apresentados ao leitor em seis teses $(01,04,05,06,09$ e 10) em capítulo específico e nas outras seis teses $(02,03,07,08,11$ e 12$)$ tais registros foram inseridos no capítulo introdutório, como item específico ou ainda no decorrer do texto.

A aproximação ao campo de pesquisa foi registrada no conjunto de trabalhados analisados. Essa aproximação ocorreu principalmente em relação à temática de estudos, mas, em geral, envolveu conversas informais por meio de informantes-chave, técnicos de esferas públicas, lideranças de movimentos sociais e espaços de trabalho. Esse registro, por exemplo, foi registrado pela Tese 05, em que "[...] contou-se com auxílio de informantes chaves já citados. Como o ingresso no local de estudo e o acesso aos estabelecimentos de agricultura familiar ocorreu basicamente através deles, esse fato facilitou e permitiu a aproximação inicial [...]" (KIRSCH, 2015, p. 113).

A abordagem qualitativa possibilita inúmeras possibilidades de técnicas para coleta de dados. Esta diversidade foi verificada no conjunto de teses aqui analisadas, entretanto, a entrevista se apresentou como a técnica predominante, pois todos os 12 trabalhos se valeram deste instrumento para geração e obtenção de dados junto aos sujeitos das investigações científicas.

Ainda em relação às entrevistas, quanto ao seu formato estrutural, os trabalhos de tese também apresentaram similaridade, pois na totalidade dos procedimentos técnicos e me- 
todológicos optou-se por entrevistas semiestruturadas ou não estruturadas. Essa perspectiva de roteiros legitima a abordagem qualitativa das construções metodológicas dos trabalhos, pois permite ao pesquisador uma maior de liberdade no desenvolver da entrevista, bem como possibilita a percepção de aspectos que não estavam previamente sob análise.

Além das possibilidades que apresentam os roteiros semi ou não estruturados, a Tese 06 registrou o procedimento de pré-teste do roteiro por meio de informantes-chave e também a realização de entrevista-teste. Este processo foi registrado pela autora: "Após a realização dessas conversas, houve as primeiras modificações no roteiro de pesquisa. Foi necessário reelaborar algumas questões, sintetizar o roteiro e submetê-lo a testes." (AG$\mathrm{NE}, 2014$, p. 66). A realização de pré-testes e entrevistas testes sem dúvida alguma se constituem em significativos avanços em termos de qualidade tanto no roteiro de entrevista, quanto para a coleta de dados.

As teses analisadas apresentaram no processo de coleta de dados a utilização de roteiros personalizados para cada grupo de sujeitos entrevistados, verificou-se este procedimento nas Teses 08 e 10. Entende-se que o objetivo dessa diferenciação decorre do fato do pesquisador procurar obter significados a partir de grupos de sujeitos com posições e olhares diferentes sobre o mesmo problema social. Nesta direção, Malhorta (2001) e Almeida e Botelho (2006) também compreendem que a formulação do roteiro de entrevista deve observar o público respondente e sua capacidade de interpretação e as condições de formular a resposta.

A observação foi outra técnica utilizada com objetivo de coleta de dados, especialmente a partir de conversas informais (fora do momento formal da realização da entrevista), observação em eventos, percepções, entre outras observações relevantes que surgem no período de estada no campo de pesquisa. Essas observações e informações foram registradas em diários de campo. Em síntese, o diário de campo foi utilizado nas Teses 02, 03, 05, 06, 08 e 09, sendo suas utilidades exemplificadas a partir das seguintes descrições:

A interação da pesquisadora com os agricultores nos mais variados ambientes e nas mais diversas situações - como, nos momentos em que as famílias estavam produzindo os alimentos, a participação em refeições, eventos e reuniões - destacou-se como um conjunto de ações pertinentes para entender os hábitos dos agricultores. Essas observações foram organizadas com o auxílio de uma ferramenta denominada diário de campo, que consiste no registro de detalhes dos dados, das observações e das reflexões [...]. (AGNE, 2014, p. 72).

Na mesma direção, Kirsch, (2015, p. 118) relata que,

Para complementar essa etapa da pesquisa e alcançar essa operacionalização, as informações, julgadas como importantes e relevantes, ou ainda, que de algum modo pudessem auxiliar na reconstrução e pintura do quadro que forma a vulnerabilidade e a resiliência, foram registradas em um diário de campo. As anotações são compostas de observações não contempladas pelo roteiro da entrevista, geralmente colhidas das conversas informais antes ou depois da sua aplicação, ou então de comentários de pessoas não entrevistadas formalmente, como outros membros da família ou vizinhos. Seu registro foi realizado geralmente ao final do dia, com o intuito de não se perder sua essência. 
A utilização de técnicas de pesquisa foi diversificada no conjunto de teses analisadas para realização deste estudo. Apesar de todas terem utilizados a entrevista, esta foi acompanhada por outra técnica de coleta de dados. Entre estas técnicas podemos ressaltar a observação, a utilização do diário de campo, os informantes-chave e, inclusive, a utilização de técnicas quantitativas, como a utilização de questionários e tratamento de dados por meio de softwares específicos de análise de dados. Essa triangulação permite complementariedade de métodos, além de possibilitar novas perspectivas relacionadas ao objeto de estudo (VERGARA, 2015).

Em relação aos procedimentos de análise de dados coletados por meio de pesquisa de campo, as teses analisadas para esta pesquisa concentram-se na análise de conteúdo (Teses 03, 04, 05, 06, 07, 09, 10 e 11), nas Teses 01 e 12 não houve o registro da realização de análise de conteúdo, entretanto, se observam traços, características de que essas se utilizaram do método de análise de conteúdo para construção das análises dos dados.

A Tese 02 implicou um estudo longitudinal se caracterizando por ser um estudo de caso por meio da abordagem qualitativa e auxiliada por elementos quantitativos (CONTI, 2016). Por fim, a Tese 08 se utilizou do conceito de racionalidade social de Weber para compreender as condutas sociais dos agricultores; a partir do paradigma teórico-interpretativo, o estudo baseou-se em uma perspectiva metodológica dos tipos ideais de Weber (BOLTER, 2013).

\section{CONSIDERAÇÕES FINAIS}

No que concerne à utilização dos métodos qualitativos de pesquisa em investigações científicas, neste trabalho buscou-se apresentar a riqueza e as mais diversas possibilidades que este método possibilita ao pesquisador. As investigações de cunho qualitativo assumem a subjetividade como parte do processo de construção do conhecimento e procuram entender os fenômenos sociais com profundidade e intensidade.

Compreende-se, também, que os métodos qualitativos se caracterizam pela não utilização de ferramental estatístico, entretanto, são dotados de inúmeros critérios de cientificidade, que impõem significativos desafios os pesquisadores qualitativos. Além da imersão no campo de pesquisa, os métodos qualitativos requerem habilidade e cuidado do pesquisador em retratar as realidades e fenômenos sociais.

A agricultura familiar se constitui em uma categoria de expressiva relevância, pois, além da questão da produção agropecuária, possui aspectos sociais, econômicos, culturais e históricos que a diferenciam de outros contextos e que caracterizam o modo de vida, produção e inserção social. Este trabalho procurou verificar quais métodos qualitativos foram utilizados no processo de investigação científica, a partir de teses selecionadas de um programa de Pós-Graduação Stricto Sensu referência no país.

Entre as principais análises, constata-se a qualidade das construções metodológicas, bem como a utilização dos métodos nos trabalhos de teses observados. As teses apresentaram diversidade na abordagem dos métodos qualitativos, embora como técnica de coleta a entrevista tenha sido utilizada pela totalidade dos trabalhos. Além da entrevista, ou- 
tras técnicas foram utilizadas para obtenção de dados no campo de pesquisa, as quais destacamos: a observação, o diário de campo, a utilização de informantes-chave.

Em relação à análise dos dados, a análise de conteúdo foi o procedimento mais utilizado. A análise de conteúdo se caracteriza pela categorização de dados em temas e organização de categorias conceituais para análise como forma de tratamento dos dados brutos. Nos trabalhos observados - em relação à análise de dados - houve ainda a ocorrência (Tese 08) de análise a partir da tipologia de Weber.

Por fim, consideramos que a construção deste estudo foi pertinente no sentido de discutir aspectos introdutórios em relação à utilização dos métodos qualitativos na temática da agricultura familiar. A diversidade de caminhos de pesquisa científica encontrada nas teses analisadas, não limita as possibilidades de investigação, antes pelo contrário, pois, as pesquisas qualitativas permitem inúmeras alternativas enquanto método. Esta diversidade de pesquisa se coloca de forma adequada, assumindo uma perspectiva de múltiplas possibilidades de investigações científicas no campo da agricultura familiar.

\section{REFERÊNCIAS}

ABRAMOVAY, Ricardo. O futuro das regiões rurais. Porto Alegre: UFRGS, 2003.

AGNE, Chaiane Leal. Mudanças Institucionais na Agricultura Familiar: as Políticas Locais e as Políticas Públicas nas Trajetórias das Famílias nas Atividades de Processamento de Alimentos no Rio Grande do Sul. 2014. Tese (Doutorado em Desenvolvimento Rural) - Universidade Federal do Rio Grande do Sul, Porto Alegre, 2014.

ALMEIDA, Alda Rosana; BOTELHO, Delane; Construção de questionários. In: ALVESMAZZOTTI, Alda Judith; GEWANDSZNAJDER, Fernando. 0 método nas ciências naturais e sociais: pesquisa quantitativa e qualitativa. 2. ed. São Paulo: Pioneira Thomson Learning, 2001.

ALVES-MAZZOTTI, Alda Judith; GEWANDSZNAJDER, Fernando. 0 método nas ciências naturais e sociais: pesquisa quantitativa e qualitativa. 2. ed. São Paulo: Pioneira Thomson Learning, 2001.

BOCCHI, Ildelbrando (Org.). Monografia para economia. São Paulo: Saraiva, 2004. BOLTER, Jairo Alfredo Genz. Interfaces e cogestão nas políticas para agricultura familiar: uma análise do Programa Nacional de Habitação Rural. 2013. Tese (Doutorado em Desenvolvimento Rural) - Universidade Federal do Rio Grande do Sul, Porto Alegre, 2013.

BRASIL. Lei 11.326, de 24 de julho de 2006. Estabelece as diretrizes para a formulação da Política Nacional da Agricultura Familiar e Empreendimentos Familiares Rurais. Diário Oficial da União, Brasília, DF, 25 jul. 2006. Seção 1, p. 1. Disponível em:

<http://www.planalto.gov.br/ccivil_03/_ato2004-2006/2006/lei//11326.htm>. Acesso em: 3 jul. 2017.

BRAYMAN, Alan. The debate about quantitative and qualitative research. In:

Quantity and quality in social research. London: Unwin Hyman, 1988.

CONTI, Irio Luiz. Organizações Sociais e Políticas Públicas: Inserção da FETRAF-SUL nas Políticas Públicas de Segurança Alimentar e Nutricional. 2016. Tese (Doutorado em De- 
senvolvimento Rural) - Universidade Federal do Rio Grande do Sul, Porto Alegre, 2016. COSTA, Francisco de Assis. Sete teses sobre o mundo rural brasileiro: antíteses. Revista da ABRA, edição especial, jul. 2013.

DEMO, Pedro. Metodologia científica em ciências sociais. São Paulo: Atlas, 1995.

DENZIN, Norman K.; LINCOLN, Yvonna S. Introdução: a disciplina e a prática da pesquisa qualitativa. In: DENZIN, Norman K.; LINCOLN, Yvonna S. (Orgs.). O planejamento da pesquisa qualitativa: teorias e abordagens. 2. ed. Porto Alegre: Artmed, 2006. p. 15-41.

GODOI, Christiane Kleinübing; BALSINI, Cristina Pereira Vecchio. A pesquisa qualitativa nos estudos organizacionais brasileiros: uma análise bibliométrica. In: GODOI, Christiane Kleinübing; BANDEIRA-DE-MELLO, Rodrigo; SILVA, Anielson Barbosa da. Pesquisa qualitativa em estudos organizacionais: paradigmas, estratégias e métodos. São Paulo: Saraiva, 2007. GODOY, Arlida Schmidt. Introdução à pesquisa qualitativa e suas possibilidades. Revista de Administração de Empresas, São Paulo, v. 35, n. 2, p. 57-63, mar./abr. 1995.

GUANZIROLI, Carlos Enrique. PRONAF dez anos depois: resultados e perspectivas para o desenvolvimento rural. Revista de Economia Rural, Rio de Janeiro, v. 45, n. 2, p. 301-328, abr./jun. 2007.

KIRSCH, Heitor Marcos. Vulnerabilidade e Resiliência Social da Agricultura Familiar do Alto Guaporé (MT) no Contexto das Mudanças Ambientais Contemporâneas. 2015. Tese (Doutorado em Desenvolvimento Rural) - Universidade Federal do Rio Grande do Sul, Porto Alegre, 2015.

KOCHE, José Carlos. Fundamentos de Metodologia Científica: teoria de ciência e iniciação à pesquisa. 20. ed. Petrópolis: Vozes, 2002.

LAPERRIÈRE, Anne. Os critérios de cientificidade dos métodos qualitativos. In: V.V.AA. A pesquisa qualitativa. Enfoques epistemológicos e metodológicos. Petrópolis-RJ: Vozes, 2008. p. 410-435.

MALHORTA, Naresh K. Elaboração de Questionários e Formulários. In: MALHORTA, Naresh K. Pesquisa de marketing. 3. ed. Porto Alegre: Bookman, 2001.

MATTEI, Lauro. Pronaf 10 anos: mapa da produção acadêmica. Brasília: MDA, 2006. (NEAD Estudos).

. Considerações acerca de teses recentes sobre o mundo rural brasileiro. RESR, Piracicaba, v. 52, Supl. 1, p. S105-S124, 2015.

MINAYO, Maria Cecilia de Souza. O desafio do conhecimento: pesquisa qualitativa em saúde. 12. ed. São Paulo: Hucitec, 2012.

MOREIRA, Vilson Alves. Educação do campo e docência no contexto da agricultura familiar: o Programa Escola Ativa (PEA/MEC) no município de Salinas - MG. 2013. Tese (Doutorado em Desenvolvimento Rural) - Universidade Federal do Rio Grande do Sul, Porto Alegre, 2013.

DMITRUK ORTIZ, Hilda Beatriz. Cadernos metodológicos: diretrizes do trabalho científico. 8. ed. rev. ampl. e atual. Chapecó, SC: Argos, 2012.

SCHNEIDER, Sergio (Org.). A diversidade da agricultura familiar. 2. ed. Porto Alegre: Editora UFRGS, 2009.

SCHWANDT, Thomas A. Três posturas epistemológicas para a investigação qualitativa: in- 
terpretativismo, hermenêutica e construcionismo social. In: DENZIN, Norman K.; LINCOLN, Yvonna S. (Orgs.). O planejamento da pesquisa qualitativa: teorias e abordagens. 2. ed. Porto Alegre: Artmed, 2006. p. 193-217.

SEYFERTH, Giralda. Campesinato e o Estado no Brasil. RAMBO, v. 17, n. 2, p. 395-417, 2011.

SILVA, Danielle. Produzindo Prerrogativas de Cidadania: o Acesso da Agricultura Familiar à Política de Fornecimento de Produtos para a Alimentação Escolar. 2015. Tese (Doutorado em Desenvolvimento Rural) - Universidade Federal do Rio Grande do Sul, Porto Alegre, 2015.

STRAUSS, Anselm L.; CORBIN, Juliet. Pesquisa qualitativa: técnicas e procedimentos para o desenvolvimento de teoria fundamentada. 2. ed. Porto Alegre: Artmed, 2008.

VERGARA, Sylvia Constant. Métodos de Pesquisa em Administração. São Paulo: Atlas, 2015.

VIEIRA, Marcelo Milano Falcão. Por uma boa pesquisa (qualitativa) em administração. In: ; ZOUAIN, Deborah Moraes. Pesquisa qualitativa em administração. Rio de Janeiro: FGV Editora, 2004.

WANDERLEY, Maria de Nazareth Baudel. Raízes do campesinato brasileiro. In: TEDESCO, João Carlos. (Org.). Agricultura familiar: realidades e perspectivas. 3. ed. Passo Fundo: EDIUPE, 2001. 